\title{
Caries dental en menores en situación de pobreza, asistidos por una fundación en Paraguay
}

\author{
Dental caries in children in poverty situation, assisted by a foundation in Paraguay \\ Diana Alicia Sanabria-Vázquez ${ }^{1, a}$, Marta Inés Ferreira Gaona ${ }^{1, b}$, Nohelia María Pérez-Bejarano ${ }^{1, b}$, Clarisse

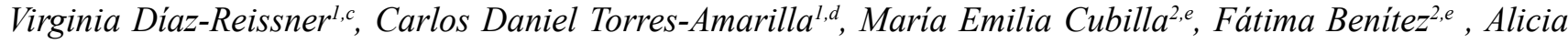 \\ Martínez $^{2, e}$, Marcelo Centurión ${ }^{2, e}$, Esteban Barrios ${ }^{2, e}$, Teresa Guerrero $^{2, e}$, Verónica Pavetti ${ }^{2, e}$
}

\section{RESUMEN}

Introducción: Si bien la salud bucal en gran parte de la población está afectada, aquella con bajo ingreso está particularmente en mayor riesgo, debido entre otros factores a la falta de acceso a atención odontológica, por el costo o falta general de información acerca de la importancia de la misma en la salud general y bienestar de las personas. Objetivos: Describir la experiencia de caries de menores en situación de calle afectados por la pobreza en la Fundación DEQUENI, sede Ypané, en el año 2013. Material y métodos: Estudio observacional descriptivo, de corte transversal, en 70 menores de 12 y 15 años de edad. Se consideraron variables demográficas, caries dental, anomalías de oclusión, y estilo de vida. Resultados: E1 54,29\% pertenecía al sexo femenino. El índice CPO-D fue 6,57 (DE=3,54). El 44,3\% de los padres afirmaron que alguna vez les enseñaron sobre salud bucal. Se informó un $100 \%$ de frecuencia de uso de cepillo dental y el $97,14 \%$ refirió cepillarse 3 veces al día. En cuanto a la dieta, el $48,57 \%$ de la población refiere al menos 5 comidas al día, el $64,29 \%$ refiere alta frecuencia de consumo de bebidas gaseosas entre las comidas o luego de la cena. Conclusiones: Los resultados ubican a esta población con una alta experiencia de caries dental y por ende una necesidad terapéutica proporcional a las mismas en concomitancia con la implementación de programas de prevención.

PALABRAS CLAVE: Caries dental, higiene bucal, factores de riesgo, Paraguay.

\footnotetext{
${ }^{1}$ Cátedra de Salud Pública, Facultad de Odontología, Universidad del Pacifico Privada. Asunción, Paraguay.

2 Facultad de Odontología. Universidad del Pacifico Privada. Asunción, Paraguay.

a Odontólogo-Cirujano.

b Especialista en Metodología de la Investigación.

c Magister en Estadística.

d Magister en Salud Pública.

e Estudiante de pregrado.
} 


\section{SUMMARY}

Introduction: Oral health affects much of the population, and the low income is particularly at risk due to lack of access to dental care, high cost of dental services and general lack of information about the importance of oral health in the general health and welfare of people. To evaluate the oral health status of children at risk affected by poverty in DEQUENI, at Ypané, in 2013. Material and methods: Observational, cross-sectional study, in 70 under 12 and 15 years old. Demographic variables, dental caries, and lifestyle were considered. Results: 54.29\% were female. The DMF-T index was $6.57(\mathrm{SD}=3,54)$. A $100 \%$ frequency of use of the toothbrush and $97.14 \%$ of brushing 3 times a day was reported. As for diet, $48.57 \%$ of the population refers at least 5 meals a day, and $64.29 \%$ reported high frequency of soft drink consumption between meals or after dinner. Conclusions: We found a high prevalence of dental caries in this population, often related to excessive consumption of foods high in sugar between meals, lack of proper hygienic habits, lack of knowledge and lack of encouragement to practice oral hygiene techniques.

\section{KEYWORDS: Dental caries, oral hygiene, risk factors, homeless youth, Paraguay}

\section{INTRODUCCIÓN}

La promoción de salud alude a la ganancia de bienestar como un todo, es la modificación de estilos de vida que conducen a la prevención de enfermedades que debe comenzar desde edades tempranas, pues es más fácil formar actitudes positivas hacia la salud en un menor que modificarlas en un adulto, pero esta situación es muy difícil en casos de menores afectados por la pobreza que salen a trabajar en edades muy tempranas, lejos de toda posibilidad de una asistencia y que se ven afectados por problemas de salud de índole diversa entre los cuales pueden mencionarse los del área bucodental (1).

En la actualidad, existen numerosas fundaciones que se ocupan de paliar esta situación, entre ellas, la Fundación DEQUENI (De Quien son los Niños), organización dedicada al apoyo sostenido de menores que trabajan en la vía pública; situación definida por la organización como de riesgo y por lo general autoexcluida del sistema de salud (por desconocimiento o falta de demanda de servicio). Brinda atención a más de 4000 niños y jóvenes de familias de escasos recursos de distintas ciudades de los departamentos Central, Cordillera y Caaguazú, que se benefician con programas a los mismos, sus familias y comunidades, para apoyar la superación de la pobreza extrema, promoviendo la autogestión y estimulando una amplia corriente de solidaridad. Están firmemente convencidos de la necesidad imperiosa de ocuparse de los menores como fundamento para construir un país diferente y mejor. Esta fundación se dedica a ayudar a madres de familia con escasos recursos brindándoles atención a los menores mientras ellas trabajan o realizan sus quehaceres diarios para que los mismos no se queden solos en la casa (2).

La salud bucal se halla afectada en gran parte de la población, pero en especial en los menores que no cuentan con conocimientos suficientes acerca de las enfermedades bucales y la manera de prevenirlas o recursos al alcance para tratarlas. Las enfermedades más frecuentes son: la caries dental, las enfermedades periodontales y maloclusiones, de las cuales la más común es la caries dental (3). Debido a que los factores de riesgo asociados a la enfermedad más común, se encuentran por lo general presentes ya en los padres, tales como como la higiene bucal deficiente; lo cual implica la presencia de placa microbiana o hábitos dietéticos incorrectos. La presencia de estos indudablemente influyen en el comportamiento y actitud de los menores según estudios realizados (4) y a su vez, estos pueden afectar el aprendizaje en la escuela y/o desempeñar cualquier trabajo, por tanto su productividad económica (5).

Las poblaciones de bajos ingresos están particularmente en riesgo debido a una complejidad de factores, entre ellos la dificultad de acceso a la atención odontológica, el elevado costo de los servicios dentales que representan y una falta general de información sobre el papel importante que desempeña la salud bucal en la salud y el bienestar de las personas $(6,7)$. Según el Ministerio de Salud Pública y Bienestar Social (MSPyBS), en su Guía de Abordaje Integral a la Salud Bucodental para el equipo de salud, en el Paraguay, el $98 \%$ de la población sufre de problemas 
de salud bucal (8). Aunque se ha mejorado el alcance de atención en salud, incluyendo el programa de atención primaria, aún podría persistir el problema de la autoexclusión ya mencionada, por lo que el mejoramiento del estado de salud bucal es incipiente.

Si todas las personas tuviesen acceso a medidas preventivas y a tratamientos adecuados y oportunos, se reduciría la presencia de enfermedades orales, se impediría su avance y la aparición de complicaciones más graves y debilitantes, y se disminuiría la necesidad de tratamientos más costosos. Esto es mucho más relevante para el caso de la salud oral, no sólo porque las enfermedades orales más comunes son prevenibles, sino porque los tratamientos pertinentes son mucho más fáciles y evitan complicaciones o tratamientos más complejos $(9,10)$.

La información obtenida servirá de base para el desarrollo de un plan de intervención acorde a las necesidades de esta población, pues no se cuenta con datos publicados de la situación de salud bucal en menores afectados por la pobreza.

El objetivo del estudio fue describir la experiencia de caries en menores afectados por la pobreza, asistidos en la Fundación DEQUENI sucursal de la ciudad de Ypané (Dpto. Central), en el año 2013.

\section{MATERIAL Y MÉTODO}

Se realizó un estudio observacional descriptivo, de corte transversal, determinando el índice CPO-D y complementando la información con la descripción de los hábitos de higiene y dieta, así como la presencia de maloclusiones. Se incluyeron a los menores de 12 y 15 años. El protocolo de investigación fue evaluado y aprobado por el Comité de Ética de la Universidad del Pacífico Privada.

La variable principal del estudio fue la experiencia de caries medida a través del Índice CPO-D y sus componentes: se consideró pieza cariada cuando la cavitación era clínicamente visible, o con presencia de obturaciones con pasta provisoria u obturaciones permanentes visiblemente filtradas. Pieza dentaria perdida: las ausentes en la cavidad bucal, por patología cariosa o muy destruidas. Pieza obturada: cuando se observó restauraciones con resina compuesta o amalgama.
Como complemento se buscó la descripción de algunos aspectos del estilo de vida, con encuesta dirigida a los padres, principalmente: Higiene bucal: Se midió la frecuencia diaria con que se realizaba. También se hicieron preguntas referentes a los elementos habitualmente utilizados en ese acto, en cuanto a la dieta: se consideró el consumo diario (entre comidas) de alimentos ricos en azúcares.

Por otra parte, la presencia de anomalías de la oclusión fue descrita con las variables: Apiñamiento: cuando las piezas dentarias superiores o inferiores estaban fuera de la línea del arco. Tipo de mordida: disgregada en las alteraciones: Borde a borde: cuando las porciones incisales antagonistas contactaban. Mordida cruzada anterior: cuando una de las piezas dentarias anterosuperiores se encontraban ocluyendo por la cara lingual de los inferiores. Mordida abierta anterior: cuando los incisivos antagonistas no contactaban. Mordida cruzada: se consideró cuando no existía contacto entre molares y premolares registrándose si era uni o bilateral.

También se tuvieron en cuenta variables demográficas como edad (12 y 15 años cumplidos) y sexo (masculino y femenino).

Todos los que cumplían con los criterios de inclusión fueron invitados a participar del estudio, constituyendo de esta manera un censo [70/80] al quedar conformado por el $87,50 \%$ de la población, [45] en niños de 12 años, [25] en niños de 15 años.

En cuanto al reclutamiento de los participantes del estudio, una vez obtenida la aprobación de las autoridades correspondientes, se organizó una reunión de padres y/o tutores, la cual fue llevada a cabo en la filial de la fundación, en la que se expuso el objetivo de la investigación y el procedimiento que se llevaría a cabo para la realización del estudio. Una vez obtenido el consentimiento informado firmado por los padres y/o responsables de los menores, se los convocó para examinar a los menores, respetando el asentimiento de los mismos.

Para la recolección de datos, se les entregó una encuesta dirigida a los los padres y/o responsables de los menores en la que se interrogó acerca de algunos factores de riesgo. La evaluación clínica se realizó en el local de la fundación, a plena luz del día, ubicando a 
los niños y jóvenes en una posición confortable, acostados sobre una superficie plana en decúbito supino con ayuda de una silla y una mesa. El examinador se dispuso por detrás del niño o niña para la obtención de una mejor visualización de la boca. El examen clínico para la determinación de la pieza dental cariada se realizó con un espejo plano sin aumento, pinza con algodón y sonda exploradora para retirar placa microbiana que impida la visibilidad.

Se realizó estadística descriptiva, utilizando frecuencia y porcentaje para variables cualitativas representados en tablas. Se aplicó estadística inferencial para comparar el CPO-D por sexo utilizando la prueba t de Student para muestras independientes, con un nivel de confianza del 95\%. También se comparó la distribución del CPO-D por grupo de edad, utilizando la prueba U de Mann-Whitney para muestras independientes con un nivel de confianza del $95 \%$, ya que no se cumplían los supuestos para pruebas paramétricas. Los programas utilizados fueron $\operatorname{Excel}^{\circ} 2013$ de $\mathrm{Mi}$ crosoft Corporation y R 3.0.0. de $R$ Foundation for Statistical Computing (11).

\section{RESULTADOS}

En el estudio fueron evaluados 70 niños de 12 y 15 años de edad. El 54,29\% eran del sexo femenino y el $64,29 \%$ tenían 12 años (Tabla 1 ).

En la evaluación del estado de salud bucodental se realizó el análisis de piezas dentales cariadas cuyos resultados fueron como sigue: los de 15 años presentaron mayor frecuencia de la enfermedad $(84,84 \%)$, en el caso de las piezas dentales perdidas la edad mayormente afectada fue la de 12 años $(27,79 \%)$. Respecto al sexo, la presencia de piezas dentales cariadas fue mayor en varones $(81,08 \%)$ y el sexo femenino se vio mayormente afectado por la cantidad de piezas dentales perdidas $(25,81 \%)$. Al comparar el CPO-D por sexo y por grupo de edad no se encontraron diferencias estadísticamente significativas. El Índice CPO-D para la población estudiada fue de 6,57 $(\mathrm{DE}=3,54)$ (Tabla 2).

E1 44,3\% de los padres afirmaron que alguna vez les enseñaron a los menores sobre salud bucal, también fueron reportados la frecuencia del uso del cepillo en

Tabla 1. Frecuencia por edad y sexo en menores de 12 y 15 años. Fundación DEQUENI, Paraguay 2013.

\begin{tabular}{ccc}
\hline Variables & No. & $\%$ \\
\hline Edad (años) & & \\
12 & 45 & 64,29 \\
15 & 25 & 35,71 \\
Sexo & & \\
Masculino & 32 & 45,71 \\
Femenino & 38 & 54,29 \\
\hline
\end{tabular}

Tabla 2. Índice COP-D en menores de 12 y 15 años. Fundación DEQUENI, Paraguay 2013.

\begin{tabular}{|c|c|c|c|c|c|c|c|c|}
\hline \multirow{2}{*}{$\begin{array}{r}\text { Variable } \\
\text { Edad }\end{array}$} & \multicolumn{2}{|c|}{ Cariados } & \multicolumn{2}{|c|}{ Perdidos } & \multicolumn{2}{|c|}{ Obturados } & \multirow{2}{*}{ CPO-D } & \multirow{2}{*}{ Sig. } \\
\hline & No. & $\%$ & No. & $\%$ & No. & $\%$ & & \\
\hline 12 años & 210 & 71,18 & 82 & 27,79 & 3 & 1,45 & $5,70 \pm 3,53$ & \multirow{2}{*}{0,567} \\
\hline 15 años & 140 & 84,84 & 24 & 14,54 & 1 & 0,6 & $6,16 \pm 3,53$ & \\
\hline \multicolumn{9}{|l|}{ Sexo } \\
\hline Masculino & 150 & 81,08 & 35 & 18,91 & 0 & 0 & $5,22 \pm 3,53$ & \multirow{2}{*}{0,135} \\
\hline Femenino & 200 & 72,72 & 71 & 25,81 & 4 & 1,45 & $6,50 \pm 3,54$ & \\
\hline
\end{tabular}


un $100 \%$, y el 97,14\% refirió cepillarse 3 veces al día.

En cuanto a dieta, la población refiere que realiza al menos 5 comidas al día en un $48,57 \%$ y que existe alta frecuencia de consumo de bebidas gaseosas entre las comidas o luego de la cena en un $64,29 \%$.

En lo que se refiere a la oclusión, en el 77,8\% de los niños de 12 años y 78,9\% de las mujeres se observó relación normal en el sector anterior, no se registró la presencia de mordida en tijera (Tabla 3 ).

El 55,55 \% del total de niños de 12 años presentaron apiñamiento dentario, al igual que el $57,84 \%$ de las mujeres (Tabla 4).

Tabla 3. Oclusión en menores de 12 y 15 años. Fundación DEQUENI, Paraguay 2013.

\begin{tabular}{|c|c|c|c|c|c|c|c|c|}
\hline & \multicolumn{4}{|c|}{ Edad (años) } & \multicolumn{4}{|c|}{ Sexo } \\
\hline & \multirow{2}{*}{\multicolumn{2}{|c|}{$\begin{array}{c}12 \\
n=45\end{array}$}} & \multirow{2}{*}{\multicolumn{2}{|c|}{$\begin{array}{c}15 \\
n=25\end{array}$}} & \multirow{2}{*}{\multicolumn{2}{|c|}{$\begin{array}{l}\text { Masculino } \\
\qquad n=32\end{array}$}} & \multirow{2}{*}{\multicolumn{2}{|c|}{$\begin{array}{c}\text { Femenino } \\
\mathrm{n}=38\end{array}$}} \\
\hline & & & & & & & & \\
\hline & No. & $\%$ & No. & $\%$ & No. & $\%$ & No. & $\%$ \\
\hline \multicolumn{9}{|c|}{ Relación Anterior } \\
\hline Normal & 35 & 77,78 & 17 & 68,00 & 22 & 68,75 & 30 & 78,95 \\
\hline $\begin{array}{l}\text { Borde a } \\
\text { borde }\end{array}$ & 6 & 13,33 & 4 & 16,00 & 4 & 12,50 & 6 & 15,79 \\
\hline $\begin{array}{l}\text { Protusión } \\
\text { maxilar }\end{array}$ & 2 & 4,44 & 4 & 16,00 & 6 & 18,75 & - & - \\
\hline $\begin{array}{l}\text { Mordida } \\
\text { cruzada }\end{array}$ & 2 & 4,44 & - & - & - & - & 2 & 5,26 \\
\hline \multicolumn{9}{|c|}{ Mordida Cruzada Posterior } \\
\hline $\begin{array}{l}\text { No dispo- } \\
\text { nible }\end{array}$ & 42 & 93,33 & 25 & 100,00 & 30 & 93,75 & 37 & 97,37 \\
\hline Unilateral & 2 & 4,44 & - & - & 2 & 6,25 & - & - \\
\hline Bilateral & 1 & 2,22 & - & - & - & - & 1 & 2,63 \\
\hline \multicolumn{9}{|c|}{ Mordida en Tijera } \\
\hline $\begin{array}{l}\text { No dispo- } \\
\text { nible }\end{array}$ & 45 & 100,00 & 25 & 100,00 & 32 & 100,00 & 38 & 100,00 \\
\hline \multicolumn{9}{|c|}{ Mordida Abierta } \\
\hline $\begin{array}{l}\text { No disponi- } \\
\text { ble }\end{array}$ & 42 & 93,33 & 24 & 96,00 & 30 & 93,75 & 36 & 94,74 \\
\hline Anterior & 3 & 6,67 & 1 & 4,00 & 2 & 6,25 & 2 & 5,26 \\
\hline \multicolumn{9}{|c|}{ Mordida Profunda } \\
\hline Ausente & 41 & 91,11 & 22 & 88,00 & 29 & 90,63 & 34 & 89,47 \\
\hline Presente & 4 & 8,89 & 3 & 12,00 & 3 & 9,38 & 4 & 10,53 \\
\hline \multicolumn{9}{|l|}{ Diastemas } \\
\hline Ausente & 36 & 80,00 & 24 & 96,00 & 26 & 81,25 & 34 & 89,47 \\
\hline Presente & 9 & 20,00 & 1 & 4,00 & 6 & 18,75 & 4 & 10,53 \\
\hline
\end{tabular}


Tabla 4. Presencia de apiñamiento dentario en menores de 12 y 15 años. Fundación DEQUENI, Paraguay 2013.

\begin{tabular}{lcccc}
\hline \multicolumn{1}{c}{ Variable } & No presenta & \multicolumn{3}{c}{ Si presenta } \\
Edad & No. & $\mathbf{\%}$ & No. & \% \\
12 años & 20 & 44,44 & 25 & 55,55 \\
15 años & 17 & 68,00 & 8 & 32,00 \\
Sexo & & & & \\
Masculino & 21 & 65,62 & 11 & 34,37 \\
Femenino & 16 & 42,10 & 22 & 57,84 \\
\hline
\end{tabular}

\section{DISCUSIÓN}

La Fundación DEQUENI, instaló un centro comunitario, el cual se encuentra en funcionamiento desde años atrás, que brinda atención a la importante población de menores existente en la ciudad de Ypané, los mismos se encuentran viviendo de manera precaria, insegura, insalubre y con escasos recursos económicos. En el presente estudio, en el mencionado centro comunitario se halló un índice CPO-D de 6,57 que representa a un nivel muy alto de la afección según el MSPyBS y la Organización Mundial de la Salud (12).

De acuerdo a estos resultados los miembros de esta comunidad no reciben atención odontológica en cuanto a prevención y tratamiento de la patología antes mencionada. Realizando una comparación con estudios realizados en poblaciones similares en la ciudad de México cuyos resultados del índice CPO-D fue de 7,3 , estos hallazgos, reflejan una brecha muy estrecha a los resultados obtenidos en el presente estudio (13); así mismo el estudio realizado en Paraguay específicamente FUNDAR (Fundación de Ayuda Republicana) también se encontró una diferencia en cuanto a la prevalencia de CPO-D que en el mismo fue de 6,2 cifra menor comparado a este estudio (14), este elevado índice indica la necesidad de atención odontológica abarcando en todas las especialidades, para el mejoramiento de la calidad de vida de los menores y sus familias. De manera a solucionar la problemática atacando los factores de riesgo, se plantearon realizar charlas educativas, haciendo énfasis sobre la importancia de la salud bucal en la salud general, enseñanza de técnicas de cepillado adecuado para cada caso en particular además de intervenciones de control y prevención y la fluorización de los dientes.
Se debe considerar que la salud bucal es parte importante de la salud general del individuo; a pesar de que las patologías bucales habitualmente no son mortales, pero representan un gran problema para la salud pública del país. El CPO-D fue mayor en mujeres; en coincidencia con la mayoría de los estudios $(13,15,16)$ y también puede ser debida al tiempo de erupción de los dientes ya que la erupción es más temprana en las niñas. La presente investigación concuerda con lo reportado por otros autores: lo que indica que a mayor edad, mayor presencia de caries dental (17).

Se debe considerar que el nivel socioeconómico y la estructura familiar podrían influir en las necesidades dentales, pues aquellos que viven en hogares con menores ingresos económicos tienen más probabilidad de necesidad de atención dental curativa. Además que, familias de escasos recursos tienen menos acceso a la información, menos recursos para comprar elementos de higiene oral y una condición desfavorable para tomar decisiones acerca del hábito dietético y acceso a la atención odontológica (18); por lo que resultaría una necesidad imperiosa aumentar el acceso a la atención dental en poblaciones de bajos ingresos y familias en situación de pobreza (19) con cobertura a problemas y necesidades de tratamiento (urgentes o no); pues privarse de tratamiento dental por su elevado costo contribuiría al acumulo de problemas de salud oral, desencadenado una deteriorada salud oral $(20,21)$

En cuanto a las características de la oclusión, en la población estudiada sólo el $55,55 \%$ del total de niños de 12 años y el $57,84 \%$ de las mujeres presentaron apiñamiento dentario, con mucho diferente de los 
niños atendidos en otra fundación paraguaya, en los que las alteraciones dento maxilares ocuparon el segundo lugar en la frecuencia de patologías afectando al $96,7 \%$ de la población total, sin reporte de diferencias por edad y sexo (14). Similar resultado fue observado en niños con caries, por Ramón Jimenez et al. (22). Resulta importante considerar este problema como factor de riesgo para el desarrollo de patologías cariosas pues a mayor apiñamiento mayor riesgo (23).

Se concluye que en esta población, hay una alta presencia de caries, es lo que establece una mayor morbilidad dentaria en los menores. Esta condición guarda relación directa con una excesiva frecuencia de consumo de alimentos ricos en azúcares entre las comidas principales, la ausencia de correctos hábitos higiénicos, falta de conocimiento y falta de estímulo para practicar las técnicas de higiene oral, de manera a mejorar su salud bucal y por consiguiente su calidad de vida. Por lo expuesto, la promoción de la salud es esencial para mejorar el estado de la salud bucal de los menores, facilitando materiales informativos y charlas educativas acerca de técnicas cepillado, las consecuencias del consumo excesivo de alimentos ricos en azúcares, la importancia de acudir al odontólogo para la detección de posibles patologías activas, etc. los cuales ayudará para mejorar la deficiencia de conocimiento existente en esta población. De igual manera atacando los factores de riesgo para disminuir el nivel de prevalencia de caries y por consiguiente la morbilidad dentaria.

\section{Correspondencia:}

\section{Dra. Clarisse Virginia Díaz Reissner. \\ Correo electrónico: diazclarisse@gmail.com}

\section{REFERENCIAS BIBLIOGRAFICAS}

1. Monroy OV, Hermosillo HV, Camacho MEI, González AM, Pérez TLS. Cambios en la prevalencia de la caries dental en escolares de tres regiones de México: encuestas de 1987-1988 y de 1997-1998. Rev Panam Salud Pública. 2003;13(5):320-6.

2. Fundación DEQUENÍ. Misión. Fernandode la Mora: Fundación DEQUENÍ; 2012. (Citado el 15 de junio del 2016) Disponible en: http://www.dequeni.org.py/ es/mision-i6

3. Juárez-López MLA, Murrieta-Pruneda JF, Teodosio -Procopio E. Prevalencia y factores de riesgo asociados a enfermedad periodontal en preescolares de la Ciudad de México. Gac Médica México. 2005;141(3):185-9.

4. Riesgo Cosme Y de la C, Costa Montané DM, Rodríguez Fernández S del C, Crespo Mafrán MI. Estado de salud bucal en escolares del Seminternado « 30 de Noviembre». Medisan. 2011;15(4):442-6.

5. Organización Panamericana de la Salud, Ministerio de Salud Pública y Bienestar Social, editores. Encuesta Nacional sobre Salud Oral Paraguay 2008. 2009. 60 p.

6. Estupiñán-Day S, Tellez M, Kaur S, Milner T, Solari A. Managing dental caries with atraumatic restorative treatment in children: successful experience in three Latin American countries. Rev Panam Salud Pública. 2013;33(4):237-43.

7. Pinilla J, Negrín-Hernández MA, Abásolo I. Time trends in socio-economic inequalities in the lack of access to dental services among children in Spain 19872011. Int J Equity Health. 2015; 14: 9. (Citado el 15 de junio del 2015) Disponible en: http://www.equityhealthj.com/content/14/1/9/abstract

8. Dirección de Salud Bucodental. Ministerio de Salud Pública y Bienestar Social. Guía de abordaje para la salud bucodental para el equipo de salud. Asunción, Paraguay: MSPBS; 2012. (Citado el 15 de junio del 2015) Disponible en: http://www.mspbs.gov.py/bucodental/wp-content/uploads/2013/04/Guia-Abordaje_enero20131.pdf

9. Barrero A, Ernesto C. Pobreza y desigualdades sociales: un debate obligatorio en salud oral. Acta Bioethica. 2006;12(1):9-22.

10. Cruz R, Caballero D, Limonta E. Nivel cognoscitivo sobre higiene bucal y gingivitis crónica en niños: Colegio «Presidente Kennedy». Venezuela. 2006. Rev Médica Electrónica. 2009; 31(4):0-0. (Citado el 15 de junio del 2015) Disponible en: http://scielo.sld.cu/scielo.php?script=sci_arttext\&pid=S1684-18242009000400007

11. Core-Team R. A Language and Environment for Statistical Computing. Vienna, Austria: R Foundation for Statistical Computing; 2014. (Citado el 15 de junio del 2015) Disponible en: http://www.R-project.org/

12. Dirección de Salud Bucodental, Ministerio de Salud Pública y Bienestar Social. Encuesta nacional sobre salud oral Paraguay 2008. Washington DC: OPS;2008.

13. Ortega-Maldonado M, Mota-Sanhua V, López-Vivanco JC. Oral health status of adolescents in México City. Rev Salud Pública. 2007; 9(3):380-7.

14. Ferreira M. Evaluation of the oral and dental health status in children on street situation, assisted by the Ayuda Republicana Foundation (FUNDAR); Paraguay: 2009-2010. Mem Inst Investig En Cienc Salud. 2011; 9(1):21-34.

15. Fernández C, Núñez L, Díaz N. Determinantes de salud oral en población de 12 años. Rev Clínica Periodoncia Implantol Rehabil Oral. 2011;4(3):117-21.

16. Cázares L, Ramos E, Tijerina L. Incremento del riesgo de padecer caries dental por consumo de hidratos 
de carbono con alto potencial cariogénico. Rev Salud Publica Nutr. 2009; 10(3):0-0. (Citado el 15 de junio del 2015) Disponible en: http://new.medigraphic.com/ cgi-bin/resumenMain.cgi?IDARTICULO=22748

17. Martínez-Pérez KM, Monjarás-Ávila AJ, Patiño-Marín $\mathrm{N}$, et al. Estudio epidemiológico sobre caries dental y necesidades de tratamiento en escolares de 6 a 12 años de edad de San Luis Potosí. Rev Invest Clín. 2010;62(3):206-13.

18. Lisboa CM, Paula JS de, Ambrosano GMB, et al. Socioeconomic and family influences on dental treatment needs among Brazilian underprivileged schoolchildren participating in a dental health program. BMC Oral Health. 2013;13(1):56.

19. Duncan L, Bonner A. Effects of income and dental insurance coverage on need for dental care in Canada. J Can Dent Assoc. 2014;80:e6.

20. Thompson B, Cooney P, Lawrence H, Ravaghi V, Quiñonez C. The potential oral health impact of cost barriers to dental care: findings from a Canadian population-based study. BMC Oral Health. 2014;14(1):78.

21. Guessous I, Theler J-M, Izart CD, et al. Forgoing dental care for economic reasons in Switzerland: a sixyear cross-sectional population-based study. BMC Oral Health. 2014; 14(1):121.

22. Ramón Jimenez R, Castañeda Deroncelé M, Corona Carpio MH, Estrada Pereira GA, Quinzán Luna AM. Factores de riesgo de caries dental en escolares de 5 a 11 años. 2016;20(5):648-55.

23. Santiesteban-Ponciano FA, Gutiérrez-Rojo MF, Gutiérrez-Rojoy JFI, Rojas-García AR. Severidad del apiñamiento dentaly su relación con la caries. 2016;46(1):29-32.

Recibido : 16/07/2015 Aceptado: 21/06/2016 